\title{
Pre-operative patient optimization to prevent postoperative pulmonary complications-Insights and roles for the respiratory therapist: A narrative review
}

\author{
Jonathon Schwartz MSE, MD1, Darian Parsey BS², Tichaendepi Mundangepfupfu MRes., MD³, Steven Tsang DO4, Robert Pranaat
} $\mathrm{MD}^{4}$, James Wilson $\mathrm{DO}^{4}$, Peter Papadakos $\mathrm{MD}^{3}$

\begin{abstract}
J Schwartz, D Parsey, T Mundangepfupfu, S Tsang, R Pranaat, J Wilson, P Papadakos. Pre-operative patient optimization to prevent postoperative pulmonary complications-Insights and roles for the respiratory therapist: A narrative review. Can J Respir Ther 2020;56:79-85. doi: $10.29390 /$ cjrt-2020-029.
\end{abstract}

\begin{abstract}
Background: The preoperative period has gained recognition as a crucial time to identify and manage preoperative medical conditions for preventing perioperative complications. Consequently, preoperative clinics have now become an essential component of perioperative care at many large hospitals. As the prevalence of preoperative clinics continues to grow, and the field of perioperative medicine progresses, respiratory therapists (RTs) will inevitably find a growing role to participate in preoperative patient optimization to mitigate pulmonary complications.

Methods: Keyword searches on perioperative pulmonary complications were conducted on the Medline database via PubMed and identified over 2000 candidate articles for review. Articles were included if they were English only and resulted with one or more of the following search terms; pulmonary complications, postoperative complications, postoperative pulmonary complications (PPCs), prehabilitation, incentive spirometry, smoking cessation, noninvasive ventilation. Preference was given for meta-analyses, randomized controlled trials, and systematic reviews. Publications within the past two decades were given additional preference toward final inclusion. The authors discussed eligible articles in group meetings over the span of multiple years to assess relevance and quality of data for narrowing eligible articles to the final selection of publications for the review.

Findings: The following narrative review examines preoperative optimization strategies to prevent PPCs and highlight areas where RTs may play a key role. After examining challenges in defining PPCs, the review examines key risk models available to predict PPCs and their implications for subsequent discussion on preventive measures that RTs may assist with in a multidisciplinary team.

Conclusion: RTs can reduce the health care burden of PPCs by assisting fellow perioperative clinicians in providing respiratory care for patients with premorbid conditions. While much of our review focused on pre-existing pulmonary pathologies and both the pharmacological and nonpharmacological optimization of these pathologies, there are other factors contributing to PPCs deserving future exploration.
\end{abstract}

Key Words: risk factors; smoking cessation; prehabilitation; noninvasive ventilation; incentive spirometry; perioperative

\section{INTRODUCTION}

Respiratory therapists (RTs) play an essential role in postoperative patient recovery and in addressing challenges to recovery due to pre-existing respiratory disease. Many medical specialties have begun to recognize the preoperative period as a pivotal opportunity to improve patient recovery and outcomes. As more and more health care specialties recognize the significance of the preoperative period, and optimizing patient comorbidities preoperatively, the respiratory therapy team will inevitably find a growing role to participate. Consequently, RTs will benefit from understanding the status on preoperative factors increasing the risk for complications and how to optimize patients preoperatively. The following narrative review will examine preoperative optimization strategies to prevent postoperative pulmonary complications (PPCs) and how RTs may play a key role. First, the review briefly defines PPCs as an introduction to examining the impact of PPCs on health care. This is followed by a discussion of established risk factors leading to PPCs, risk models for PPCs, key topics in pulmonary pathophysiology leading to
PPCs, and the role of preoperative planning for postoperative care and how it relates to the common interventions employed by RTs. Finally, the paper focuses on "prehabilitating" patients with common pulmonary diseases preoperatively and the many unanswered questions in the field of pulmonary prehabilitation.

\section{METHODS}

Keyword searches on perioperative pulmonary complications were conducted on the MEDLINE database via PubMed and identified over 2000 candidate peer-reviewed publications. Articles were included if they were English only and resulted with one or more of the following search terms; pulmonary complications, postoperative complications, PPCs, prehabilitation, incentive spirometry, smoking cessation, noninvasive ventilation (NIV). Preference was given for meta-analyses, randomized controlled trials, and systematic reviews. Case series and reports were also considered eligible for inclusion. Publications within the past two decades were given additional preference toward final inclusion.

\footnotetext{
${ }^{1}$ Department of Anesthesiology, Stony Brook University Hospital, Stony Brook, NY, USA

${ }^{2}$ Stony Brook School of Medicine, Stony Brook, NY, USA

${ }^{3}$ Department of Anesthesiology $\mathcal{E}$ Perioperative Medicine, Division of Critical Care Medicine, University of Rochester Medical Center, Rochester, NY, USA

${ }^{4}$ Department of Anesthesiology $\mathcal{E}$ Perioperative Medicine, University of Rochester Medical Center, Rochester, NY, USA

Correspondence: Jonathon Schwartz, Department of Anesthesiology, Stony Brook University Hospital, Health Sciences Center, Level 4, Stony Brook, NY 11794, E-mail: Jonathon.Schwartz@stonybrookmedicine.edu
}

Published online at https://www.cjrt.ca on 4 December 2020 
The authors discussed eligible articles in group meetings over the span of multiple years to assess relevance and quality of data for narrowing eligible articles to the final selection of publications for the review. Ethical approval was not obtained for this narrative review of published literature.

\section{FINDINGS}

What Defines PPCs? Challenges and Considerations

One may identify PPCs as unintended changes in lung function that could impair a patient's postoperative recovery. In some cases, the PPCs may present acutely with fulminant respiratory failure; however, in other cases the PPCs may present more insidiously. For example, the respiratory therapist might notice a patient is requiring more bronchodilator treatments, showing subtle increases in respiratory effort, or an increasing need for support with NIV; these may all portend clinical deterioration. Definitions for PPCs should be precise and objective to classify an event of clinical significance that is associated with a risk for impaired postoperative recovery. Events of clinical significance are inextricably tied to increased health care costs, health care quality, and patient suffering.

PPCs cover a wide range of clinical events and conditions. Across the literature, outcome definitions for PPCs can range from a singular event to more comprehensive composite measures. A 2006 study by the American College of Physicians demonstrated that many studies (60\% of 16 studies) utilized a composite outcome of pneumonia and respiratory failure to define PPCs in a patient $[1,2]$. Significant variability in definitions remain, despite the relatively recent release of European Perioperative Clinical Outcome (EPCO) definitions [1]. These definitions are provided below in Table 1 with their respective diagnostic challenges and considerations, while Table 2 provides additional PPCs that fall outside of the EPCO definitions.

Demarcating when the postoperative period starts creates an additional challenge in defining PPCs. For example, does the postoperative period start at the first extubation attempt upon completion of the surgery or should the period start only after a successful extubation? Many studies on PPCs lack a specified start point. Postponing the start timepoint for PPCs could impact estimates of incidence, and potentially misdirect attention away from earlier postoperative events that depend on preoperative optimization strategies.
Furthermore, demarcating the end time for the postoperative period also becomes a concern. As the sampling period extends from days to weeks after surgery, the influence of postoperative care systems contributes more toward the variability in the incidence of PPCs. Additionally, some definitions for PPCs require an intervention, like mechanical ventilation, or a symptom, such as a cough, to exceed a threshold duration. In such cases, rapidly reversible events may inappropriately be excluded. The difficulties outlined above serve to both question the definition of PPCs and to impact the reader's analysis of the study outcomes and interventions in the following literature and statistical models.

\section{Overall Incidence of PPCs}

The overall incidence of PPCs varies widely due to a multitude of factors including, but not limited to, the study's definition of PPCs, the operation being performed, and the patients' comorbidities. For example, a multicenter observational study published in 2015 reported an incidence of PPCs at $9.7 \%$ for their Chinese inpatient cohort [3]. These were patients undergoing noncardiac surgery with general or regional anesthesia. Conversely, a recent study indicated an incidence of $24.6 \%$ in patients following elective craniotomy under general anesthesia [5]. McAlister et al. [6] showed that $2.7 \%$ of patients who presented to the University of Alberta pre-admission clinic between 2001 and 2003 developed PPCs within 7 days of their surgery. Because of all the heterogeneity in types of surgery and other variables, we will focus on a general patient population that probably estimates a relevant incidence of 10\% [7].

\section{The Impact of PPCs}

PPCs incur added hospitalization time, health care costs and portend increased morbidity and mortality that may extend years beyond the perioperative period $[1,7,8]$. Several studies suggest a wide range of cost increase ranging from $\$ 25,000$ to $\$ 180,000$ (USD) [9]. These estimates of added costs reveal the economic burden from PPCs that may far exceed hospital cost for a routine surgery. Development of PPCs has also been shown to prolong length of stay (LOS) by an average of 4.5 days in one study and may reach as high as 13-17 additional days [9]. Variability in LOS estimates likely depend on definitions and study population, with more severe types of PPCs likely associating with more LOS prolongation. Development of PPCs may also be associated with an increased mortality rate. Estimates on postoperative mortality with PPCs increased

\section{TABLE 1}

European Perioperative Clinical Outcome definitions for perioperative pulmonary complications

\begin{tabular}{lll}
\hline Complication & Definition & Ease of diagnosis/reproducibility \\
\hline Respiratory infection & $\begin{array}{l}\text { Patient has received antibiotics for a suspected respiratory infection and met } \\
\text { one or more of the following criteria: new or changed sputum, new or } \\
\text { changed lung opacities, fever, white blood cell count }>12 \times 10^{9} / \mathrm{L}\end{array}$ & $\begin{array}{l}\text { Easy, measurable lab values and imaging for } \\
\text { diagnosis }\end{array}$
\end{tabular}

Respiratory failure

Pleural effusion

Pneumothorax

Bronchospasm

Aspiration pneumonitis
Postoperative $\mathrm{PaO}_{2}<8 \mathrm{kPa}(60 \mathrm{mmHg})$ on room air, a $\mathrm{PaO}_{2}: \mathrm{Fl02}$ ratio $<40$ $\mathrm{kPa}(300 \mathrm{mmHg})$ or arterial oxyhemoglobin saturation measured with pulse oximetry $<90 \%$ and requiring oxygen therapy

Chest radiograph demonstrating blunting of the costophrenic angle, loss of sharp silhouette of the ipsilateral hemidiaphragm in upright position, evidence of displacement of adjacent anatomical structures or (in supine position) a hazy opacity in one hemithorax with preserved vascular shadows

Source: Adapted from Miskovic and Lumb et al. [1]
Lung opacification with a shift of the mediastinum, hilum or hemidiaphragm toward the affected area, and compensatory over-inflation in the adjacent nonatelectatic lung

Air in the pleural space with no vascular bed surrounding the visceral pleura

Newly detected expiratory wheezing treated with bronchodilators

Acute lung injury after the inhalation of gastric contents
Difficult, variability in $\mathrm{SpO}_{2}$ targets/hypoxia definition for initiating supplemental oxygen

Difficult, possible confounder of human error due to discrepancy interpretation, e.g., subjectivity of "hazy"

Difficult, mild findings are subtle with less reproducibility compared to significant or lobar involvement

Easy, often obvious in setting of hypoxia

Difficult, lacking consistent standard to measure against

Easy, history of episode of aspiration prior to injury 
TABLE 2

Additional definitions of Postoperative pulmonary complications that are not present in European Perioperative Clinical Outcome definitions

\begin{tabular}{|c|c|c|}
\hline Complication & Definition & Ease of diagnosis/reproducibility \\
\hline Hypoventilation/hypercapnic respiratory failure & $\begin{array}{l}\text { Elevation of the arterial carbon dioxide tension, } \\
\text { seen in hypoventilation }\end{array}$ & $\begin{array}{l}\text { Easy, detected clinically with bradypnea, rapid } \\
\text { shallow breathing, and altered mentation and } \\
\text { confirmed readily with blood gas analysis }\end{array}$ \\
\hline $\begin{array}{l}\text { Hypoxic respiratory failure mixed hypercapnic and } \\
\text { hypoxic respiratory failure }\end{array}$ & $\begin{array}{l}\text { Postoperative } \mathrm{PaO}_{2}<8 \mathrm{kPA}(60 \mathrm{~mm} \mathrm{Hg}) \text { on } \\
\text { room air, a } \mathrm{PaO}_{2}: \mathrm{FIO} 2 \text { ratio }<40 \mathrm{kPA}(300 \mathrm{mg} \\
\mathrm{Hg}) \text {, or arterial oxyhemoglobin saturation } \\
\text { measured with pulse oximetry }<90 \% \text { and } \\
\text { requiring oxygen therapy }\end{array}$ & $\begin{array}{l}\text { Easy, detected with clinical criteria, oximetry, } \\
\text { and blood gas analysis }\end{array}$ \\
\hline ARDS/TRALI & $\begin{array}{l}\text { Ventilated, bilateral infiltrates on CXR, } \\
\mathrm{PaO}_{2}: \mathrm{FIO}_{2}<300 \mathrm{mg} \mathrm{Hg} \text {, minimal evidence of } \\
\text { left atrial fluid overload within } 7 \text { days of surgery }\end{array}$ & $\begin{array}{l}\text { Easy, set criteria and CXR findings with no } \\
\text { cardiac cause }\end{array}$ \\
\hline Pulmonary embolism & $\begin{array}{l}\text { Obstruction of the pulmonary artery or one of its } \\
\text { branches by material from elsewhere in the } \\
\text { body }\end{array}$ & $\begin{array}{l}\text { Difficult in patients with low-intermediate } \\
\text { pretest risk requiring CT angiography and } \\
\text { exclusion of other prevalent causes }\end{array}$ \\
\hline Pulmonary edema & $\begin{array}{l}\text { Pulmonary congestion/hypostasis, acute edema } \\
\text { of the lung, CHF, fluid overload }\end{array}$ & $\begin{array}{l}\text { Difficult, imaging required with subjective } \\
\text { interpretation }\end{array}$ \\
\hline Exacerbation of pre-existing pulmonary disease & Not further defined & Easy, patient past medical history \\
\hline
\end{tabular}

Note: ARDS, Acute Respiratory Distress Syndrome; CHF, Congestive Heart Failure; CXR, Chest X-Ray; TRALI, Transfusion Related Acute Lung Injury.

to $11.2 \%$ from the baseline $0.7 \%$ after bowel surgery. Another investigation found a similar 30-day mortality rate approaching 14\%-30\% for patients with PPCs, versus $0.2 \%-3 \%$ for patients without PPCs $[1,7]$. The impact of PPCs on mortality, however, may extend as much as 5 years following surgery and could reflect definitions for PPCs in studies that consist of only more severe types of PPCs [1].

\section{Risk Factors for PPCs}

In a large database review of risk factors for PPCs, age was found to be the second most identified factor throughout the 11 studies [2]. The site of surgery also had a significant impact on the likelihood of developing PPCs, notably that the percentage of patients that developed PPCs during hospitalization for peripheral, abdominal, and intrathoracic procedures was $5.8 \%, 23.0 \%$, and $51.3 \%$, respectively [4]. These factors can be considered nonmodifiable and while they should be kept in mind during an initial assessment, there are other factors that a multidisciplinary team can influence to reduce PPCs.

Many patients have comorbidities that predispose to the development of PPCs and thus present opportunities for intervention. Chronic obstructive pulmonary disease (COPD) is a frequent cause of pulmonary complications, with an OR of 1.79 (95\%, 1.44-2.22) when eight studies were pooled and analyzed [2]. A history of asthma similarly increased the incidence of PPCs from $4.8 \%$ to $8.7 \%$ [10]. While not a chronic illness, the presence of a respiratory infection within 1 month of surgery drastically increased the incidence from $4.2 \%$ to $17.8 \%$ [10].

Two risk factors deserve separate attention because of their potential ease of use as screening tools for RTs and physicians alike. First, McAlister et al. [6] validated previous data stating that a positive preoperative cough test was found to be independently associated with increased PPCs (OR 3.8). A positive preoperative cough test was defined as additional unintended coughs after the test subject performs a single forced cough following deep inspiration. Additionally, Canet et al. [10] found preoperative $\mathrm{SpO}_{2}$ when breathing room air to be the strongest patient related PPC risk factor. In total, $3 \%$ of patients with $\geq 96 \% \mathrm{SpO}_{2}, 9.8 \%$ of patients with $91-95 \% \mathrm{SpO}_{2}$, and $28.6 \%$ of patients with $\leq 90 \% \mathrm{SpO}_{2}$ developed a PPC. These are easily measured, objective findings that the respiratory therapist can use to quickly assess a patient's risk at the bedside. Of note, it is not known if improving the $\mathrm{SpO}_{2}$ preoperatively reduces the risk associated with these decreased levels.

Risk Stratification Across Surgical Populations

Readers exploring the literature on risk factors for PPCs will notice shared factors across models derived from different populations, definitions for PPCs, and study outcomes.
In 2006, a systematic review was conducted exploring clinical and laboratory predictors of PPCs in general surgical patient populations that would help guide internists with preoperative consultations [2]. Patient-related risk factors including advanced age (OR 2.09-3.04), ASA classification >2 (OR 2.55 - 4.87), CHF (OR 2.93), COPD (OR 1.79), and functional dependency $(1.65-2.51)$ had good evidence for predicting PPCs [2].

The Assess Respiratory Risk in Surgical Patients in Catalonia (ARISCAT) trial [10] expanded model applicability through a prospective, multicenter study of nearly 2500 patients undergoing elective or emergent surgery. A seven-variable regression model was developed to separate patients into low-, intermediate-, and high-risk groups for PPCs according to the EPCO definition for PPCs [11]. Of note, four risk factors, namely low preoperative pulse oximetry, recent respiratory tract infection, age, and low hemoglobin concentration accounted for $55 \%$ of a patient's risk for suffering PPCs. The ARISCAT model is of interest as it was subsequently externally validated by the PERISCOPE (prospective evaluation of a risk score for postoperative pulmonary complications in Europe) trial [12]. In PERISCOPE [12], the researchers tested the geographic portability of the ARISCAT model across 63 centers in Europe. A total of 725 PPCs were recorded for 404 (7.9\%) of 5099 total patients. Predicted versus observed rates of PPCs for low, intermediate, and high risk were $3.39 \%$ (ARISCAT score <26), $12.98 \%$ ( $\geq 26$ and $<45$ ), and $38.01 \%$ ( $\geq 45)$, respectively, with a c-statistic of 0.89 .

After validating the ARISCAT model on an external dataset, the PERISCOPE study group subsequently turned their attention toward development of a new predictive model. They conducted a secondary analysis of the PERISCOPE dataset encompassing 5384 patients across 63 hospitals in Europe [13]. Postoperative respiratory failure was defined as a partial pressure of oxygen in arterial blood $\left(\mathrm{PaO}_{2}\right)$ less than $8 \mathrm{kPa}$ $(60 \mathrm{mmHg})$ or a new onset $\mathrm{SpO}_{2}$ less than $90 \%$ while breathing room air that required oxygen therapy, noninvasive, or invasive mechanical ventilation. Independent predictors included: low preoperative peripheral $\mathrm{SpO}_{2}$, at least one preoperative respiratory symptom, chronic liver disease, congestive heart failure, intrathoracic or upper abdominal surgery, procedure duration exceeding $2 \mathrm{~h}$, and emergency surgery.

Yepes-Temino et al. [14] in 2016 studied 559 patients undergoing lung resection in a retrospective multi-center observational study evaluating for external validation of the Clinical Prediction Rule for Pulmonary Complications (CPRPCs). They found that the CPRPC model, initially devised for primary lung cancer resection, lacked discriminatory power in the broader study cohort, so Yepes-Temino et al. [14] derived a new predictive model. The novel multivariate predictive model evaluated the impact of a multitude of risk factors. Upon model regression fitting, only 
age, smoking status, and predicted postoperative $\mathrm{FEV}_{1} \%$ attained statistical significance and an overall discriminatory performance. Despite the new model's improved performance, the scope of the model remains limited to thoracic surgery.

From the above studies, there are several common risk factors for PPCs surface, notably advanced age, ASA classification, surgery duration and location, preexisting respiratory disease, and a low preoperative $\mathrm{SpO}_{2}$. A summary of risk factors organized by study and similarity is provided in Table 3.

\section{Treatment of PPCs}

Early therapeutic options for patients that are at risk of developing PPCs are available and effective at improving the patient's clinical course. These include early mobilization of the patient and adequate pain management to allow full return of respiratory muscle function. Physiotherapists performing preoperative education on deep diaphragmatic breathing exercises and airway clearance techniques, followed by early mobilization (in bed activity, sitting, standing, walking) as soon as tolerated postoperatively, has been shown to reduce the rate of postoperative pulmonary atelectasis [16]. Much controversy exists over the use of incentive spirometry (IS), a similar treatment strategy to deep breathing, as a prophylactic measure against pulmonary complications in surgical patients. There is wide variability in data regarding IS use, potentially because of implementation differences among providers. Hospitals and their departments prescribe different breaths per hour, sessions per day, and methods of use, all of which may lead to different outcomes. Amidst this variation in practice, the 2011 American Association for Respiratory
Care (AARC) guidelines state that IS should not be used independently to prevent PPCs, including atelectasis. The guideline authors agree that IS does, however, remain a potential therapeutic tool if used with deep breathing techniques, early mobilization, and appropriate analgesic management [17].

If the patient develops PPCs despite prophylactic care, PPC treatment depends on which specific PPCs each individual patient is most likely to have given their current circumstances. For example, the patient with likely pneumonia would need an antibiotic regimen targeting the likely pathogen. Likewise, in the hypoxemic patient, there is a natural progression in therapy from oxygen via nasal cannula to mechanical ventilation via an endotracheal tube. Depending on the severity of hypoxemia, nasal canula or facemask ventilation can be used, which is followed by NIV if the patient does not improve. Use of high flow nasal canula therapy as opposed to standard oxygen supplementation has been a growing practice for noninvasive positive pressure in the treatment of PPC's secondary to atelectasis, suspected pneumonia, and hypercapnia $[18,19]$. Patients with progressing respiratory insufficiency despite these measures may require endotracheal intubation. Invasive mechanical ventilation should be used for the shortest duration possible with attempts at liberation initiated as soon possible. In summary, increasing severity of PPCs understandably calls for more intensive treatments, which culminates when required in postoperative mechanical ventilation.

It seems reasonable to suspect that many of the interventions above that are targeted for the postoperative period may also assume a greater role in the preoperative period for prehabilitation programs. Improving ambulation and initiating continuous positive airway pressurization

TABLE 3

Postoperative pulmonary complications predictive models

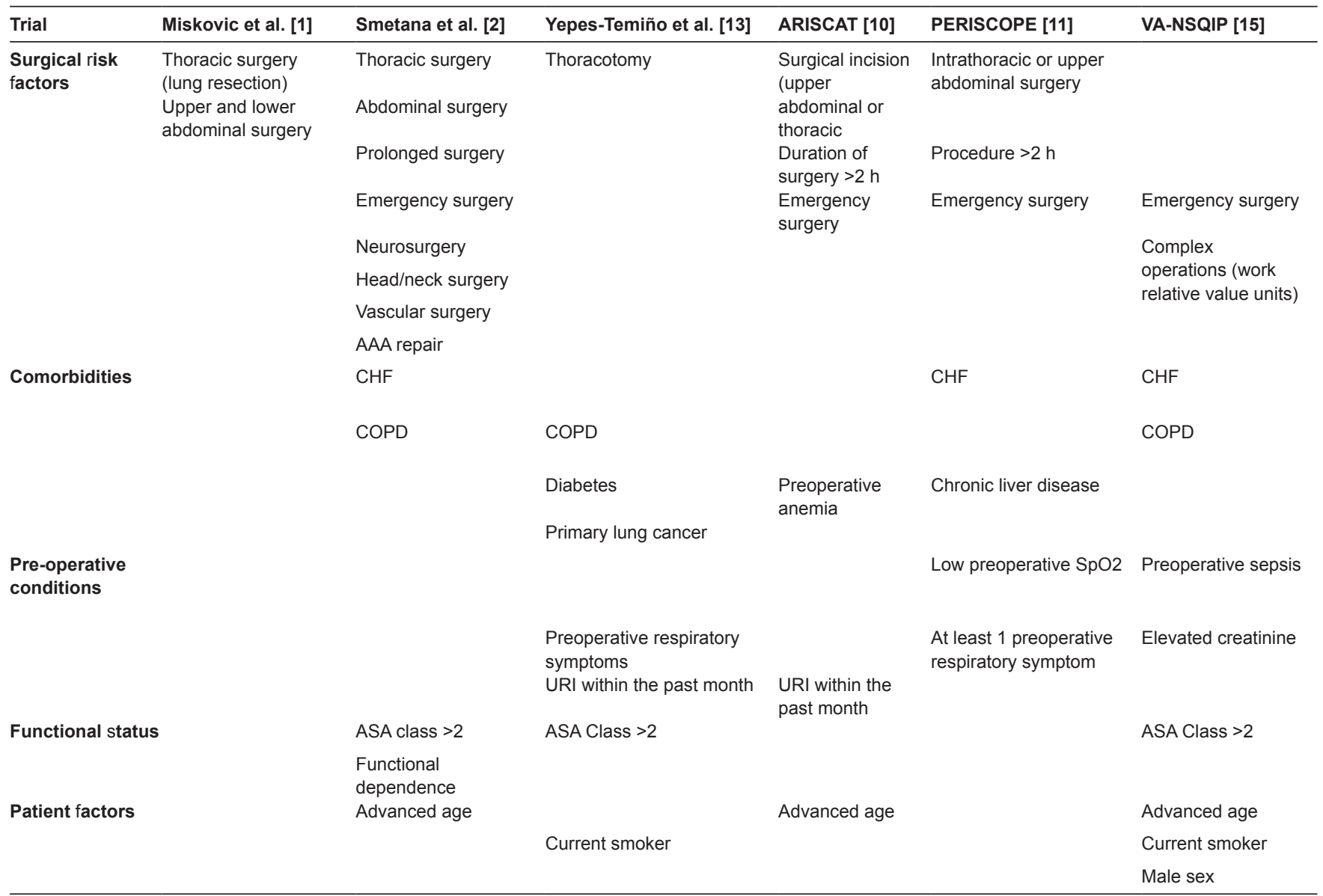

Note: AAA- Abdominal Aortic Aneurysm, ASA Class- American Society of Anesthesiologists Physical Status Classfication, CHF- Congestive Heart Failure, COPD- Chronic Obstructive Pulmonary Disease, $\mathrm{SpO}_{2}$ - Blood Oxygen Saturation levels, URI- Upper Respiratory Infection. 
(CPAP) preoperatively are gaining more attention and popularity as preoperative interventions. Additionally, as perioperative care coordination improves through clinical pathways (e.g., enhanced recovery after surgery, perioperative surgical home), the role for the RT to participate in patient education of breathing exercises and equipment (e.g. IS, CPAP mask) is likely to increase. RTs may also play a key role in supervising and designing educational programs and systems for the preoperative care team.

\section{Can Pulmonary Prehabilitation Reduce PPCs}

Underpinning successful pulmonary prehabilitation is medical optimization of a patient's comorbidities, especially conditions such as those found within risk stratification models. For example, with the available evidence, best practice appears to be continuing all asthma or COPD medications perioperatively to avoid adverse outcomes [20].

Systematic reviews of strategies to prevent PPCs after noncardiothoracic surgery have identified only a few successful interventions [21, 22]. Prehabilitation is the method of optimizing physical functionality before an operation with the intent of improving a patient's postoperative status. Pulmonary prehabilitation is any exercise, device, or procedure that is introduced preoperatively to prevent or reduce the risk of postoperative respiratory decline. This can vary from psychosocial behavioral changes implemented months before surgery (i.e., smoking cessation) to IS and respiratory muscle strengthening in the patient's room. Below is a list of common prehabilitation techniques available to RTs.

Noninvasive ventilation for obesity and obstructive sleep apnea (OSA)

In a recent qualitative review and meta-analysis of obese adults (body mass index $>35 \mathrm{~kg} / \mathrm{m}^{2}$ ) receiving perioperative NIV, Carron et al. [22] found that patients benefited from fewer postoperative pulmonary complications. Specifically, when these patients received NIV $>5$ min prior to and following surgery, their $\mathrm{PaO}_{2}$ values were significantly increased. Postoperative NIV was also associated with improved forced vital capacity. Notably, NIV did not achieve statistical significance in the prevention of reintubation or unplanned intensive care unit admission. However, the number needed to treat to prevent one PPC was $11.3 \mathrm{~A}$ randomized control trial assessed the efficacy of NIV in addition to standard therapy in 50 obese patients undergoing bariatric surgery. There were reduced events of both pneumonia and atelectasis with NIV usage [23]. These findings imply that utilization of preoperative NIV and a structured plan for delivering NIV postoperatively can prevent PPCs in the increasing population of obese surgical patients. This supports the consensus that pre- and postoperative NIV reduces pulmonary dysfunction and promotes recovery [24].

Obesity is a common risk factor for the development of OSA. The Society of Anesthesia and Sleep Medicine guidelines examine the role for preoperative CPAP therapy among diagnosed OSA patients adherent to CPAP, diagnosed patients nonadherent to CPAP, and surgical patients with a high probability of OSA. The key studies reviewed by the guidelines committee demonstrating a beneficial effect on global postoperative outcome with CPAP therapy applied preoperatively and/or postoperatively can be found in the published guidelines article (table 8 in [25]. The task force recommends involvement of practitioners with expertise in sleep medicine as studies have shown improved adherence. Although the data on how preoperative knowledge of polysomnography results and compliance records impact perioperative outcomes are lacking, the committee recommended that obtaining the data would be meaningful to patient care.

Interestingly, a recent study in lobectomy patients that assessed for multiple PPCs found no statistically significant reduction in any outcome when preoperative NIV was compared with control [26]. While this should not lead to the abolishment of NIV use, it calls for future research and more careful patient selection for NIV treatment. Compiling the findings from this study in lobectomy patients with the above studies, one may surmise that in OSA and obese patients that the discrepancies on NIV impact could stem from heterogenous study patient populations, NIV duration, programmed pressures, and patient compliance among other factors.
Smoking cessation

Smoking is a well-known risk factor for causing both general and pulmonary complications after surgery and is a risk factor for PPCs. The physiologic stress associated with anesthesia and surgery places a significant demand on the cardiopulmonary system. Cigarette smoking places an additional physiologic stress on the respiratory system by increasing mucus production, impairing ciliary secretion clearance, and increasing small airway reactivity via inflammation [27]. These factors may result in inadequate oxygen delivery to vital organs, thereby compromising overall postoperative recovery [28].

Smoking cessation has an established evidence base for preventing PPCs [27]. The harmful effects of smoking on postoperative outcomes is well known and was recently validated in a large observational study reaffirming that smokers had increased morbidity, mortality, and respiratory complications [29]. Smoking cessation at least 8 weeks prior to surgery has been shown to reduce PPCs [30]. Smoking cessation within 8 weeks of surgery, however, has shown an increase in postoperative complications. Patients undergoing coronary artery bypass graft, who refrained from smoking within 8 weeks of surgery experienced a fourfold increase in PPCs compared with those who stopped greater than 8 weeks prior to surgery. This paradoxical increase in PPCs may be due in part to an increase in airway reactivity. Despite these data, the optimal timing of preoperative smoking cessation remains poorly defined [31].

A 2014 Cochrane review searched for randomized studies enrolling patients who smoked and were awaiting any type of planned surgery [32]. The trials tested interventions including nicotine replacement therapy (NRT), varenicline therapy, brief face-to-face interventions, and more extended behavioral interventions to assist with preoperative smoking cessation. The review found evidence that preoperative smoking interventions providing behavioral support and NRT were associated with an increased short-term smoking cessation and reduced postoperative morbidity. Interventions that started four to eight weeks before surgery were more likely to have an impact on complications and on long-term smoking cessation.

Recent data support the claim that smoking cessation at any time preoperatively improves postoperative outcomes; 462 patients undergoing lung resection for nonsmall cell lung cancer were evaluated on their smoking status. The patients were separated into two groups, having quit $>6$ weeks or $<6$ weeks prior to surgery. Both groups showed reduced incidence of PPCs compared with patients who currently smoked; however, there were no outcome differences between the groups. Additionally, there was no difference in long-term survival with a median follow-up time of 29.8 months [33].

Preoperative smoking cessation through a structured program may offer significant impact with modest resource requirements. Implementation of a preoperative smoking cessation program at St. Joseph's Hospital in London, Ontario, resulted in a significant decrease in smoking rates on the day of surgery and at 30-day follow-up. Interestingly, the overall rate of combined intraoperative and immediate postoperative complications did not differ between groups though the PPCs were not assessed in isolation between the groups. A preoperative screening questionnaire was conducted at least 3 weeks before surgical dates. Those patients found to have active cigarette use were given a brief, 5-min counseling session by trained preoperative clinic nurses. Additionally, informational brochures on smoking cessation, as well as a 6-week nicotine replacement supply dosed to number of cigarettes used daily, were provided to the patients. The preoperative clinic would then issue a referral to the Canadian Cancer Society's Smoker's Helpline with the helpline pursuing up to 4 attempts at initiating contact with patients and if contact made proceed with at least 4 contact sessions per patient to facilitate smoking cessation [3].

Exercise and conditioning: physiotherapy, exercise, incentive spirometry In a multi-center randomized pragmatic trial of patients undergoing abdominal surgery, Boden et al. [15], studied the difference that a 30-min preoperative physiotherapy education and breathing exercises made in reducing PPCs within 14 days following surgery or hospital 
discharge, with an absolute risk reduction of $15 \%$ and a NNT of 7 . Pasquina et al. [34] speculated that this effect is likely more pronounced when education and breathing exercise training occurs prior to surgery instead of following surger. A Cochrane database review of 12 trials with a total of 695 participants showed reduction in atelectasis and pneumonia postoperatively when preoperative inspiratory muscle training was done (RR 0.53, 95\% CI 0.32-0.82 and RR 0.45, 95\% CI 0.26-0.77, respectively) [34]. In addition, Katsura et al. [35] reported a reduction in the LOS. In this study, inspiratory muscle training consisted of strength and endurance training 5-7 times per week for at least 2 weeks before surgery. These data show that preoperative intervention and management by RTs can reduce PPCs.

\section{The Future of RT in Preventing PPCs Before Surgery}

RTs can play a critical role in the preoperative optimization of patients with the goal of prompt and effective pulmonary recovery in the postoperative period. Awareness of current risk models and evidence-based prevention strategies can help RTs perform the best care when called to help prepare a patient for surgery.

The perioperative surgical home refers to a patient-centered care model where the patient's surgical experience is overseen by a multidisciplinary team focused on maintaining integrated care throughout the surgical episode. The respiratory therapist will ultimately find a role within the surgical home by providing more input and care for patients in the preoperative period prior to surgery as prehabilitation and smoking cessation initiatives continue to expand. Finding the team design and workflow remains a rich territory for future research.

\section{CONCLUSION}

In summary, RTs can reduce the health care burden of PPCs by assisting fellow perioperative clinicians in providing respiratory care for patients with premorbid conditions. While much of our review focused on pre-existing pulmonary pathologies and both the pharmacological and nonpharmacological optimization of these pathologies, there are other factors contributing to PPCs deserving future exploration.

\section{Summary of Recommendations}

- Definitions of a PPC vary widely across studies and readers should exercise caution before comparing changes in PPC incidence between two different studies

- A positive cough test and low preoperative $\mathrm{SpO}_{2}$ can be useful indicators of increased PPC risk.

- Advanced age, ASA classification, surgery duration, location, and preexisting respiratory disease are among the most widely accepted risk factors for PPCs.

- While IS has controversial benefit, we believe it remains useful when paired with pain management, early mobilization, and deep breathing exercises.

- Smoking cessation prior to surgery decreases rates of PPCs, but an ideal time period of cessation has yet to be firmly defined

- RT-guided patient education and exercise could improve patient outcomes by decreasing PPC incidence and LOS.

\section{DISCLOSURES}

\section{Contributors}

JS contributed toward literature research, conceptual organization, writing, editing, and review of the final manuscript; DP contributed toward literature research, content organization, writing, editing, and review of the final manuscript; TM contributed toward content organization, editing, and review; ST contributed toward literature research, initial drafting, and final review; RP contributed toward literature research, initial drafting, and final review; JW contributed toward literature research, initial drafting, and final review; PP is responsible for the conception of the manuscript topic, approval of conceptual organization of the manuscript, and both editing and review of the manuscript. All authors reviewed and approved the final manuscript.

\section{Funding}

The authors have no funding sources to disclose with this manuscript

\section{Competing Interests}

All authors have completed the ICMJE uniform disclosure form at www. icmje.org/coi_disclosure.pdf and declare: no financial relationships with any organizations that might have an interest in the submitted work in the previous 3 years; no other relationships or activities that could appear to have influenced the submitted work.

\section{Ethics Approval}

Ethics approval was not pursued for this narrative review manuscript.

\section{REFERENCES}

1. Miskovic A, Lumb AB. Postoperative pulmonary complications. BJA Br J Anaesth 2017;118(3):317-34. doi: 10.1093/bja/aex002.

2. Smetana GW, Lawrence VA, Cornell JE. Preoperative pulmonary risk stratification for non cardiothoracic surgery: systematic review for the American College of Physicians. Ann Intern Med 2006;144:581-95. doi: 10.7326/0003-4819-144-8-200604180-00009.

3. Lee SM, Landry J, Jones PM, Buhrmann O, Morley-Forster P. The effectiveness of a perioperative smoking cessation program. Anesthes Analges 2013;117(3):605-13. doi: 10.1213/ANE.0b013e318298a6b0.

4. Jin $\mathrm{Y}, \mathrm{Xie} \mathrm{G}$, Wang $\mathrm{H}$, et al. Incidence and risk factors of postoperative pulmonary complications in noncardiac Chinese patients: a multicenter observational study in University Hospitals. BioMed Res Int 2015;2015:1-10. doi: 10.1155/2015/265165.

5. Sogame LCM, Vidotto MC, Jardim JR, Faresin SM. Incidence and risk factors for postoperative pulmonary complications in elective intracranial surgery. J Neurosurg 2008;109(2):222-7. doi: 10.3171/ JNS/2008/109/8/0222.

6. McAlister FA, Bertsch K, Man J, Bradley J, Jacka M. Incidence of and risk factors for pulmonary complications after nonthoracic surgery. Am J Respir Crit Care Med 2005;171(5):514-17. doi: 10.1164/rccm. 200408-1069OC.

7. Agostini P, Cieslik H, Rathinam S, Bishay E, Kalkat MS, Rajesh PB, et al. Postoperative pulmonary complications following thoracic surgery: are there any modifiable risk factors? Thorax 2010;65(9):815-18. doi: 10.1136/thx.2009.123083.

8. Sachdev G, Napolitano LM. Postoperative pulmonary complications: pneumonia and acute respiratory failure. Surg Clin NA 2016;92(2):32144. doi: 10.1016/j.suc.2012.01.013.

9. Fleisher LA, Linde-Zwirble WT. Incidence, outcome, and attributable resource use associated with pulmonary and cardiac complications after major small and large bowel procedures. Perioper Med (London, England) 2014;3:7. doi: 10.1186/2047-0525-3-7.

10. Canet J, Gallart L, Gomar C, Paluzie G, Vallès J, Castillo J, et al. Prediction of postoperative pulmonary complications in a population-based surgical cohort. Anesthesiology 2010;113(6):1338-50. doi: 10.1097/ALN.0b013e3181fc6e0a.

11. Paluzie G, Valle J, Castillo J, Ph D, Sabate S. Prediction of postoperative pulmonary complications in a population-based surgical cohort. Anesthesiology 2010;113(6):1338-50. doi: 10.1097/ALN.0b013e3181 fc6eOa.

12. Mazo V, Sabaté S, Canet J, Gallart L, De Abreu MG, Belda J, et al. Prospective external validation of a predictive score for postoperative pulmonary complications. Anesthesiology 2014;121(2):219-31. doi: 10.1097/ALN.0000000000000334.

13. Abreu MGD, Belda J, Canet J, Sabate S. Development and validation of a score to predict postoperative respiratory failure in a multicentre European cohort a prospective, observational study. Eur J Anaesthesiol 2015;32(7):458-70. doi: 10.1097/EJA.0000000000000223.

14. Yepes-Temiño MJ, Monedero P, Pérez-Valdivieso JR, Grupo Español de Anestesia T. Risk prediction model for respiratory complications after lung resection. Eur J Anaesthesiol 2016;33(5):326-33. doi: 10.1097/ EJA. 0000000000000354 .

15. Boden I, Skinner EH, Browning L, Reeve J, Anderson L, Hill C, et al. Preoperative physiotherapy for the prevention of respiratory complications after upper abdominal surgery: pragmatic, double blinded, multicentre randomised controlled trial. BMJ 2018;360:55916. doi: 10.1136/ bmj.j5916.

16. Hanada M, Kanetaka K, Hidaka S, Taniguchi K, Oikawa M, Sato S, et al. Effect of early mobilization on postoperative pulmonary complications in 
patients undergoing video-assisted thoracoscopic surgery on the esophagus. Esophagus 2018;15(2):69-74. doi: 10.1007/s10388-017-0600-x.

17. Restrepo RD, Wettstein R, Wittnebel L, Tracy M. Incentive spirometry: 2011. Respiratory Care 2011;56(10):1600-4. doi: 10.4187/respcare.01471.

18. Ferrando C, Puig J, Serralta F, Carrizo J, Pozo N, Arocas B, et al. Highflow nasal cannula oxygenation reduces postoperative hypoxemia in morbidly obese patients: a randomized controlled trial. Minerva Anestesiol 2019;85(10):1062-70. doi: 10.23736/S0375-9393.19.13364-0.

19. Wu X, Cao W, Zhang B, Wang S. Effect of high-flow nasal cannula oxygen therapy vs conventional oxygen therapy on adult postcardiothoracic operation. Medicine 2018;97(41):e12783. doi: 10.1097/MD.0000000000012783.

20. Renew JR, Aniskevich S. Perioperative pulmonary medication management. Curr Clin Pharmacol 2017;12(3):182-7. doi: 10.2174/157488471266617091 8150757.

21. Va L, Je C, Gw S. Clinical guidelines. Strategies to reduce postoperative pulmonary complications after noncardiothoracic surgery: systematic review for the American College of Physicians. Ann Intern Med 2006;144(8):596-40.13p. doi: 10.7326/0003-4819-144-8-200604180-00011.

22. Carron M, Zarantonello F, Tellaroli P, Ori C. Perioperative noninvasive ventilation in obese patients: a qualitative review and meta-analysis. Surg Obes Relat Dis 2016;12(3):681-91. doi: 10.1016/j.soard.2015.12.013.

23. Cavalcanti MGDO, Andrade LB, Santos PCPD, Lucena LRR. Noninvasive preventive ventilation with two pressure levels in the postoperative period of roux-en-y gastric bypass: randomized trial. Arq Bras Cir Dig 2018;31(1):e1361. doi: 10.1590/0102-672020180001e1361.

24. Perrin C, Jullien V, Vénissac N, Berthier F, Padovani B, Guillot F, et al. Prophylactic use of noninvasive ventilation in patients undergoing lung resectional surgery. Respir Med 2007;101(7):1572-8. doi: 10.1016/j. rmed.2006.12.002.

25. Chung F, Memtsoudis SG, Ramachandran SK, Nagappa M, Opperer M, Cozowicz C, et al. Society of anesthesia and sleep medicine guidelines on preoperative screening and assessment of adult patients with obstructive sleep apnea. Anesth Analg 2016;123(2):452-73. doi: 10.1213/ ANE.0000000000001416.

26. Paleiron N, Grassin F, Lancelin C, Tromeur C, Margery J, Natale C, et al. Assessment of pre-operative noninvasive ventilation before lung cancer surgery: the preOVNI randomized controlled study. J Thorac Cardiovasc Surg 2019;160(4):1050-1059.e3. doi: 10.1016/j.jtcvs.2019.09.193.
27. Theadom A. Effects of preoperative smoking cessation on the incidence and risk of intraoperative and postoperative complications in adult smokers: a systematic review. Tobacco Contr 2006;15(5):352-8. doi: 10.1136/tc.2005.015263.

28. Delgado-Rodriguez M, Medina-Cuadros M, Martínez-Gallego G, Gómez-Ortega A, Mariscal-Ortiz M, Palma-Pérez S, et al. A prospective study of tobacco smoking as a predictor of complications in general surgery. Infect Control Hosp Epidemiol 2003;24(1):37-43. doi: 10.1086/502113.

29. Yuce TK, Khorfan R, Soper NJ, Hungness ES, Nagle AP, Teitelbaum EN, et al. Post-operative complications and readmissions associated with smoking following bariatric surgery. J Gastrointest Surg 2020;24(3):52530. doi: 10.1007/s11605-019-04488-3.

30. Warner MA, Offord KP, Warner ME, Lennon RL, Conover MA, Jansson-Schumacher U. Role of preoperative cessation of smoking and other factors in postoperative pulmonary complications: a blinded prospective study of coronary artery bypass patients. Mayo Clin Proc 1989;64(6):609-16. doi: 10.1016/S0025-6196(12) 65337-3.

31. Mason DP, Subramanian S, Nowicki ER, Grab JD, Murthy SC, Rice TW, et al. Impact of smoking cessation before resection of lung cancer: a society of thoracic surgeons general thoracic surgery database study. Ann Thorac Surg 2009;88(2):362-71. doi: 10.1016/j.athoracsur.2009.04.035.

32. Thomsen T, Villebro N, Møller AM. Interventions for preoperative smoking cessation. Cochrane Database Syst Rev 2014;(3):CD002294-CD. doi: 10.1002/14651858.CD002294.pub4.

33. Lugg ST, Tikka T, Agostini PJ, Kerr A, Adams K, Kalkat MS, et al. Smoking and timing of cessation on postoperative pulmonary complications after curative-intent lung cancer surgery. J Cardiothor Surg 2017;12(1):52. doi: 10.1186/s13019-017-0614-4.

34. Pasquina P, Tramér MR, Granier J-M, Walder B. Respiratory physiotherapy to prevent pulmonary complications after abdominal surgery. Chest 2006;130(6):1887-99. doi: 10.1378/chest.130.6.1887.

35. Katsura M, Kuriyama A, Takeshima T, Fukuhara S, Furukawa TA. Preoperative inspiratory muscle training for postoperative pulmonary complications in adults undergoing cardiac and major abdominal surgery. Cochrane Database Syst Rev 2015;(10):CD010356. doi: 10.1002/14651858.CD010356.pub2. 